\title{
Field-induced motion of nematic disclinations
}

\author{
PaOlo Biscari ${ }^{1}$ and Timothy J. Sluckin ${ }^{2}$ \\ ${ }^{1}$ Dipartimento di Matematica, Politecnico, Piazza Leonardo da Vinci 32, 20133 Milano (Italy) and \\ Istituto Nazionale di Fisica della Materia. Via Ferrata 1 - 27100 Pavia (Italy). \\ ${ }^{2}$ Faculty of Mathematical Studies, University, Southampton SO17 1BJ (United Kingdom).
}

Date: 9 November 2018

2000 MSC: 76A15 - Liquid crystals

\begin{abstract}
An individual defect in a nematic liquid crystal moves not only in response to its interaction with other defects but also in response to an external field. We analyze the motion of a wedge disclination in the presence of an applied field of strength $H$. We neglect backflow and seek steadily travelling patterns. The stationary picture yields a semi-infinite wall of strength $\pi$, bounded by the defect line. We find that the disclination advances into the region containing the wall at velocity $v(H)$, where $v$ scales as $H /|\log H|$ as long as the magnetic coherence length is greater than the core radius. When the external field is applied in the presence of a pair of disclinations, their dynamics is strongly influenced. We compute the expected relative velocity of the disclinations as a function of distance and field. The natural tendency for the disclinations to annihilate each other can be overcome by a sufficiently strong field suitably directed.
\end{abstract}




\section{Introduction}

Singularities in liquid crystals, or defects, have played a fundamental role not only in the development of the understanding of the physics of liquid crystals but also in the later development of the topological theory of defects in condensed matter. In nematic liquid crystals, point, line and wall defects can be found. Line defects were first classified by F.C. Frank, who noted that line defects came in classes with an integer or half-integer charge 1]. Later workers, using the topological theory of defects, realised that nematic liquid crystals sustain a topologically unique line defect [2] 3. 4. However, Frank's naïve classification, which effectively supposes that the nematic order parameter is restricted to a plane, remains important in providing guidance and intuition for the physics of defects in nematic liquid crystals.

The topological total charge of a system is conserved during its evolution. Under many circumstances this remains true for Frank's definition of charge; this is a stronger condition. For instance, defects of opposite charges may annihilate and defects of higher topological charges may decay to a bunch of defects of smaller topological charges. Topological dipoles may even be nucleated from a smooth field 5 .

In this paper we focus on nematic disclinations, i.e. line defects. According to Frank's definition, the topological charge of a line defect is defined as the number of turns the director performs along a closed path surrounding the defect. This number may be half-integer because of the headand-tail symmetry of nematic liquid crystal molecules. When the final director is rotated by an angle $\pi$ with respect to the initial director, the physical state they describe does not exhibit any discontinuity.

The physical manifestation of the topological theory of defects in nematic liquid crystals concerns escape into the third dimension. Nematic disclinations of integer charge are not topologically stable. A suitable continuous transformation, involving the third previously neglected dimension, dissolves the singularity and leaves behind a regular field [6]. In a similar manner, all half-integral disclinations can be distorted into each other. But often free energy criteria impede this process, and the Frank classification remains useful. In particular, annihilation of opposite charge half-integral defects is favored, but of same-charge defects is hindered.

Defect dynamics has been widely studied either in the classical Oseen-Zocher-Frank (OZF) theory [7, 8, 1, or within the extended de Gennes-Ericksen theory [9, 10]. However, there are fundamental dilemmas in treating defect motion. The OZF theory is not a dynamical theory, and defect cores are regions in which, strictly speaking, the OZF theory does not apply. The theory can be extended to deal with moving defects, but only by introducing a phenomenological dissipation function using director rate of change. This minimal extension is incorrect; the full extension involves hydrodynamic terms which depend on the local director [11. But the local director is not defined in the defect region, and so this extension is also inadequate to describe defect motion. The alternative de Gennes-Ericksen approach (now usually called the $\mathbf{Q}$-tensor theory) is in principle up to the task, but now the defect regions are no longer special; there is a danger of confusing calculation with computation, leading to answers of doubtful physical significance.

Despite these problems, some theoretical progress has been made, in part because some authors have detected analogous topological structures in liquid crystals and cosmological models. A single disclination may move through an otherwise smooth field [12], but then the problem is why the defect is moving in the first place. An implicit response to this question is to give more attention to the interaction between two or more defects. Now the defects move, slowly, to reduce their elastic energy. For example, attraction between two point defects of opposite charges has been studied both in planar [13, 14, 15] and cylindrical [16, 17, 18, geometries. The attraction between a dipole of smectic disclinations has been studied in [19.

More precise quantitative descriptions of the defect evolution must necessarily take into account backflow effects [20, 21, 22], i.e. the interactions between director rotation and macroscopic molecular motion. The first analytical attempt to introduce backflow effects was performed in [23, where the macroscopic velocity field was coupled to the degree of orientation, though not to the director field. A series of recent numerical simulations [24, 25, 26, 27] have determined the main effects of backflow coupling. The dynamical director patterns turn out to be strongly 
influenced in the final part of the annihilation process, but some effects may be noted even during all the defect evolution. In particular, the positive-charge disclination moves almost twice as faster than the negative-charge one [26].

In this paper we investigate the effect of applied external field on the defect dynamics. We consider a simple geometry: a single $+\frac{1}{2}$, or a dipole of $\pm \frac{1}{2}$ disclinations, in planar symmetry. We compute the defect speed with the aid of the Leslie's dissipation balance [11. It turns out that the external field exerts a profound effect on the defect dynamics and the defect interaction. By suitably adjusting the external field strength and direction it is possible to drive a single disclination through the sample, almost at will. More interestingly, the external field may not only accelerate the annihilation process. It can also stop the defects, or even reverse their velocities, so transforming an attractive into a repulsive interaction. Depending on the external field direction, a critical defect distance may arise. This characterizes the defect interaction. They annihilate each other if they come closer than that distance; otherwise, they repel.

Throughout our presentation we will introduce and discuss some assumptions that simplify our analytical calculations. The 1-constant approximation in the elastic free energy, and a parabolic approximation in the magnetic energy linearize the free energy derivatives. We remark that the parabolic approximation should be abandoned if we were to generalize the present study to highly charged defects. We also neglect backflow. This approximation allows us to derive an analytical condition which determines the defect velocity, and in particular the critical distance that reverses the defect interaction. A numerical analysis would correct these computed values, even if the described phenomena will certainly remain.

The plan of the paper is as follows. In Section 2 we analyse the motion of a single disclination in an external field. Section 3 describes the defect interaction, and how it can be reversed with the aid of the applied field. Section 4 briefly summarizes and discusses our results.

\section{Single defect motion}

We consider a $+\frac{1}{2}$ disclination embedded in an external magnetic field. This field will favor the director orientation of one side of the defect with respect to the other side. This asymmetry is sufficient, as we shall see, for energy considerations alone to determine that the defect will move, and to determine the direction of its motion. Our task is to determine the magnitude of the velocity as a function of the field intensity. We adopt the 1-constant approximation and neglect backflow. This latter approximation implies that our estimated velocity will be certainly smaller than the actual velocity. In fact, backflow effects reduce the total dissipation, so allowing faster director dynamics.

Let us consider the reference frame illustrated in Figure 10 It moves with the disclination, which sits at the origin $O$. The $x$-axis is parallel to the external field. Given any point $P$ in the plane, let $\vartheta$ be the polar angle between the radial direction $(P-O)$ and the $x$-axis. Disclinations of half-integer charge do not benefit from escaping into the third dimension. Thus, we restrict our interest to situation in which the director $\mathbf{n}$ is confined to the plane, and let $\Phi(P)$ be the angle the nematic director at $P$ determines with the $x$-axis.

In the absence of a field, the 1-constant approximation implies that the director angle varies linearly with the polar angle: $\Phi=\frac{1}{2} \vartheta$. In the presence of the field the same linear dependence holds, roughly speaking, on length scales smaller than the magnetic coherence length $\xi$. So long as we confine ourselves to these length scales, the elastic energy overwhelms the external field. However, far from the defect, the magnetic energy forces the system toward its preferred value $\Phi=0$. On the other hand, over a closed path around the defect, no matter how far that path may be from the defect, topology forces the angle $\Phi$ to rotate through $\pi$. This creates a dilemma, for the system must rotate through an angle $\pi$, and yet remain at $\Phi=0$.

These two constraints are reconciled by restricting the region over which $\Phi$ rotates. This region is a topologically irreducible wall between regions of space whose director is oppositely oriented. Associated with the wall is a well-defined surface free energy analogous to the surface tensions of fluid mechanics. This is a $\pi$-wall, since the director concentrates its $\pi$-rotation in it. In the 


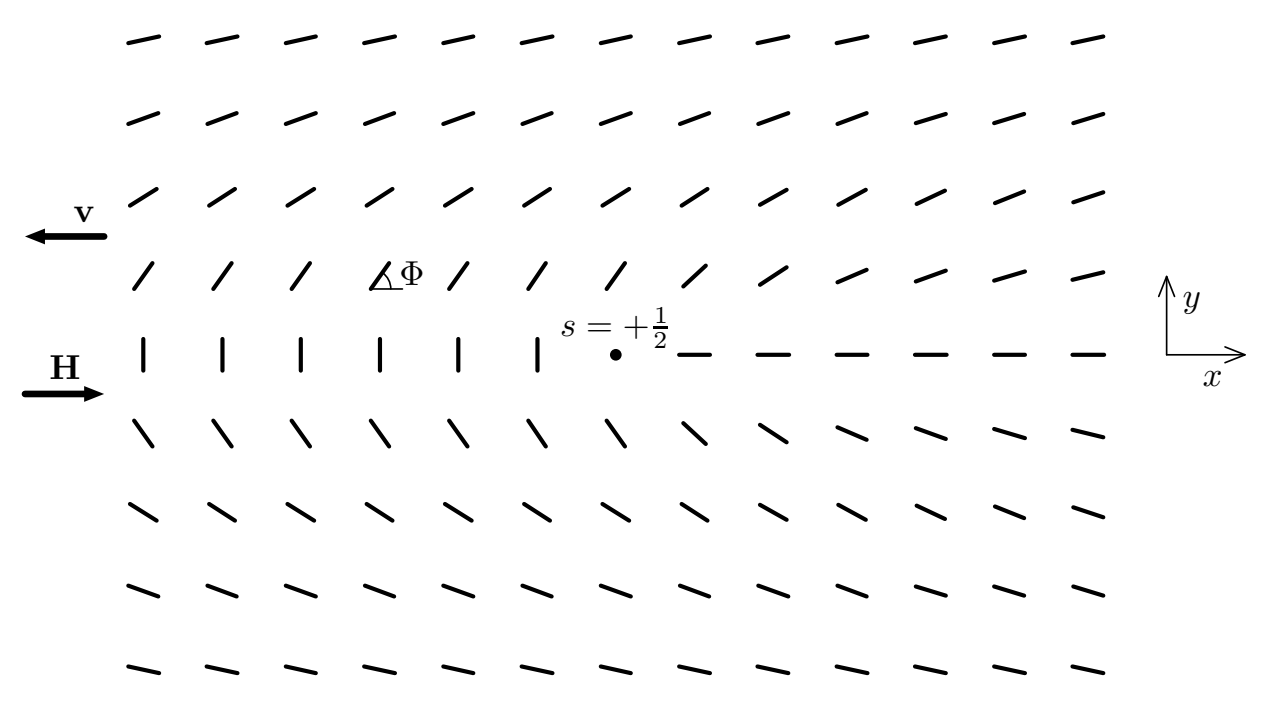

Figure 1: Geometric setting for the analysis of the single defect motion. The defect occupies the origin of the co-moving reference frame, and its moves towards the $\pi$-wall. The angle $\Phi$ is determined by the director and the motion direction (orthogonal to the external field).

presence of the field, the previously isolated defect line has been transformed into the trailing edge of a wall defect. The disclination then moves into the wall in order to reduce the wall area and consequently the free energy of the system.

\subsection{Dissipation principle}

We determine both the director field and the value of the disclination velocity by imposing the energy balance between the free-energy loss-rate and the dissipation [11:

$$
\dot{\mathcal{F}}+\mathcal{D}=0 \text {. }
$$

In the 1-constant approximation, the free-energy functional is given by

$$
\mathcal{F}[\Phi]=\int_{\mathbb{R}^{2}}\left(K|\nabla \Phi|^{2}+\chi_{\mathrm{a}} H^{2} \sin ^{2} \Phi\right) d a,
$$

where $K$ is an average elastic constant, $\chi_{\mathrm{a}}$ is the magnetic anisotropy, and $H$ is the strength of an external magnetic field.

It is well-known that a $+\frac{1}{2}$ disclination possesses an infinite core energy. The elastic free-energy density diverges at the defect, and the singularity is not integrable. There are two ways to avoid this divergence. The first consists in excluding from our integration region a small disk centered in the defect, the core region $\mathcal{B}_{\circ}$. The radius of the excised disk, the core radius $r_{\circ}$, is usually much smaller than all other characteristic lengths entering the problem, so that many studies have been performed in the limit of vanishing $r$ [28. A more precise physical description of the defect requires an extension of the classical Oseen-Zocher-Frank theory, and the replacement of the nematic director with the nematic order tensor [29]. We will choose the first option, and perform all the integrations in the pierced domain, which excludes $\mathcal{B}_{\circ}$. We further assume that the core radius is fixed. The basic physics of the phenomenon is well-described using these approximations. However, in order to deal with external fields of any intensity, it would be interesting to apply the techniques of 30. to determine how and when the magnetic coherent length influences the core radius.

A second, though less worrying, free-energy divergence comes from the supposedly infinite size of the domain. The domain may extend indefinitely in the $y$-direction without inducing any 
singularity, since both the elastic and the magnetic energy densities vanish away from the $x$-axis. On the contrary, there will be few cases in which a large- $x$ cut will be needed to keep energies finite. In those cases we will assume that our domain is bounded by $|x| \leq L$. Whenever possible, we will perform the $L \rightarrow+\infty$ limit, and we will notice that the large scale length $L$ will not finally enter in the defect velocity.

Our final approximation concerns the magnetic free-energy. We will perform the parabolic approximation $\sin ^{2} \Phi \simeq \Phi^{2}$. This approximation allows us to obtain linear field equations in $\Phi$. It can be used in the whole domain provided we define $\Phi \in\left[-\frac{\pi}{2}, \frac{\pi}{2}\right)$, since it that case all the values attained by the director angle belong to the potential well of the equilibrium configuration $\Phi=0$. We remark that this approximation would not be valid if we were interested in analysing the equilibrium configurations of more complex defects.

When we neglect backflow, the dissipation assumes the simple expression

$$
\mathcal{D}=\gamma_{1} \int_{\mathcal{B}_{\circ}} \dot{\Phi}^{2} d x d y
$$

where the pierced integration domain $\mathcal{B}_{\circ}$ comes into play since also the dissipation density diverges in the core region.

Let us perform an infinitesimal displacement $\delta \Phi$ of the director field. If we make use of the divergence theorem, the dissipation principle (2.1) requires

$$
\int_{\partial \mathcal{B}_{\circ}} \delta \Phi(2 K \nabla \Phi) \cdot \nu d \ell+\int_{\mathcal{B}_{\circ}} \delta \Phi\left[\gamma_{1} \dot{\Phi}-2 K \Delta \Phi+2 \chi_{\mathrm{a}} H^{2} \Phi\right] d a=0 \quad \forall \delta \Phi,
$$

where $\nu$ is the outer normal along $\partial \mathcal{B}_{\circ}$. The arbitrariness of $\delta \Phi$ requires that the quantity in square brackets in the second integral must vanish identically:

$$
\gamma_{1} \dot{\Phi}=2 K \Delta \Phi-2 \chi_{\mathrm{a}} H^{2} \Phi .
$$

Equation (2.5) is the well-known time-dependent Ginzburg-Landau evolution equation of the system. It will supply us the director field. However, the Ginzburg-Landau equation alone is not able to guarantee the dissipation principle. We must check that also the first integral in (2.4) vanishes. The boundary $\partial \mathcal{B}_{\circ}$ is made of two parts: a small circle around the defect and a large boundary at infinity. The integral around the latter vanishes since at infinity $\nabla \Phi$ fades everywhere, except along the $\pi$-wall, where it is orthogonal to the outward normal. We are then left with the first integral in (2.4), performed along the boundary of the core region. This quantity has a simple physical meaning [31]: it is the power supplied by the core region to the rest of the domain. Thus, to require that all the free-energy loss is dissipated within the system is equivalent to require that no energy is supplied to it, neither from the outside nor from the core region.

\subsection{Steadily moving defects}

We will look for stationary solutions of equation (2.5). They aim at representing a defect moving at a constant speed $v$ towards the $\pi$-wall. We will find that, for any positive value of $v$, it is possible to find a solution of (2.5) which satisfies the boundary conditions. However, there is just one value of the velocity which cancels also the first integral in (2.4) (or, equivalently, that satisfies the global dissipation condition). It is the only velocity at which the defect is able to move without any external boost.

In a steadily moving reference frame, traveling with the defect itself, the relative stationary differential equation follows by replacing in (2.5) the time derivative with $v \partial_{x}$. We thus obtain:

$$
\Delta \Phi-\frac{\lambda}{\xi} \frac{\partial \Phi}{\partial x}-\frac{\Phi}{\xi^{2}}=0
$$

where $\xi:=\sqrt{\frac{K}{\chi_{\mathrm{a}} H^{2}}}$ is the magnetic coherence length, and $\lambda:=\frac{\gamma_{1}}{2 \sqrt{K \chi_{\mathrm{a}}}} \frac{v}{|H|}$ is a dimensionless quantity. We look for solutions of the eigenvalue problem (2.6) that satisfy the symmetry 
requirement $\Phi(x,-y)=-\Phi(x, y)$ for all $y \neq 0$, and the boundary conditions

$$
\Phi\left(x, 0^{+}\right)=\left\{\begin{array}{ll}
0 & \text { if } x>0 \\
\frac{\pi}{2} & \text { if } x<0,
\end{array} \quad \text { and } \quad \lim _{y \rightarrow \infty} \Phi(x, y)=0 \quad \text { for all } x \in \mathbb{R},\right.
$$

where the latter condition is determined by the presence of the magnetic field. The boundary conditions 2.7) are singular only at the origin, where a disclination of topological charge $+\frac{1}{2}$ stands. Indeed, the discontinuity the angle $\Phi$ suffers along the negative $x$-axis does not induce any physical singularity, since $\Phi=\pi / 2$ and $\Phi=-\pi / 2$ describe the same director orientation.

Among the solutions of the eigenvalue problem (2.6)-(2.7), the dissipation principle (2.1) will single out the only physical one.

\subsection{Director field}

We solve the linear partial differential equation (2.6) with a Fourier transform. To this aim, it is useful to write the first of the boundary conditions (2.7) as

$$
\Phi\left(x, 0^{+}\right)=\frac{\pi}{4}-\frac{1}{4 \mathrm{i}} \mathrm{PV} \int_{\mathbb{R}} \frac{\mathrm{e}^{\mathrm{i} q x}}{q} d q \quad \text { for all } \quad x \neq 0,
$$

where PV denotes the Cauchy Principal Value of an integral. We then look for solutions of equation (2.6) of the form:

$$
\Phi(x, y)=\frac{\pi}{4} g_{1}(y)-\frac{1}{4 \mathrm{i}} \mathrm{PV} \int_{\mathbb{R}} \frac{\mathrm{e}^{\mathrm{i} q x}}{q} g_{2}(q, y) d q,
$$

with

$$
g_{1}(0)=g_{2}(q, 0)=1 \quad \text { and } \quad \lim _{y \rightarrow \infty} g_{1}(y)=\lim _{y \rightarrow \infty} g_{2}(q, y)=0 \quad \text { for all } q \in \mathbb{R} .
$$

If we insert (2.9) in (2.6) we obtain:

$$
\frac{\pi}{4}\left(g_{1}^{\prime \prime}-\frac{1}{\xi^{2}} g_{1}\right)-\frac{1}{4 \mathrm{i}} \mathrm{PV} \int_{\mathbb{R}} \frac{\mathrm{e}^{\mathrm{i} q x}}{q}\left[\frac{\partial^{2} g_{2}}{\partial y^{2}}-k^{2}(q) g_{2}\right] d q=0,
$$

where $k(q)$ will henceforth denote the positive-real-part square-root of $k^{2}(q)=q^{2}+\frac{\mathrm{i} q \lambda}{\xi}+\frac{1}{\xi^{2}}$. The solution of (2.11) and (2.10) in the upper half-plane $\{y \geq 0\}$ is

$$
\Phi(x, y)=\frac{\pi}{4} \mathrm{e}^{-\frac{y}{\xi}}-\frac{1}{4 \mathrm{i}} \mathrm{PV} \int_{\mathbb{R}} \frac{\mathrm{e}^{\mathrm{i} q x-k(q) y}}{q} d q,
$$

whereas, by symmetry, the solution in the whole plane is given by:

$$
\Phi(x, y)=\varepsilon(y)\left[\frac{\pi}{4} \mathrm{e}^{-\frac{|y|}{\xi}}-\frac{1}{4 \mathrm{i}} \mathrm{PV} \int_{\mathbb{R}} \frac{\mathrm{e}^{\mathrm{i} q x-k(q)|y|}}{q} d q\right],
$$

with

$$
\varepsilon(y):=\left\{\begin{aligned}
1 & \text { if } y \geq 0 \\
-1 & \text { if } y<0
\end{aligned}\right.
$$

Figure 2 illustrates the solution (2.13). More precisely, in the upper plot it is possible to observe how the magnetic free-energy density is concentrated on the $\pi$-wall, whereas the lower plot shows that the elastic free-energy density is mostly localized on the defect. In both cases, it is possible to notice that the decay pattern from $\Phi= \pm \frac{\pi}{2}$ on the left $x$-axis to the equilibrium value $\Phi=0$ becomes constant when we move some magnetic coherence lengths away from the defect.

We notice that no use of the core region has been made for the time being. In fact, the differential problem (2.6)-(2.7) admits a solution in $\mathbb{R}^{2} \backslash(0,0)$, without any need of excising a finite region around the defect. We will see below that this will not be the case when we will have to deal with the derivatives of the field, and in particular with the dissipation (2.3). 


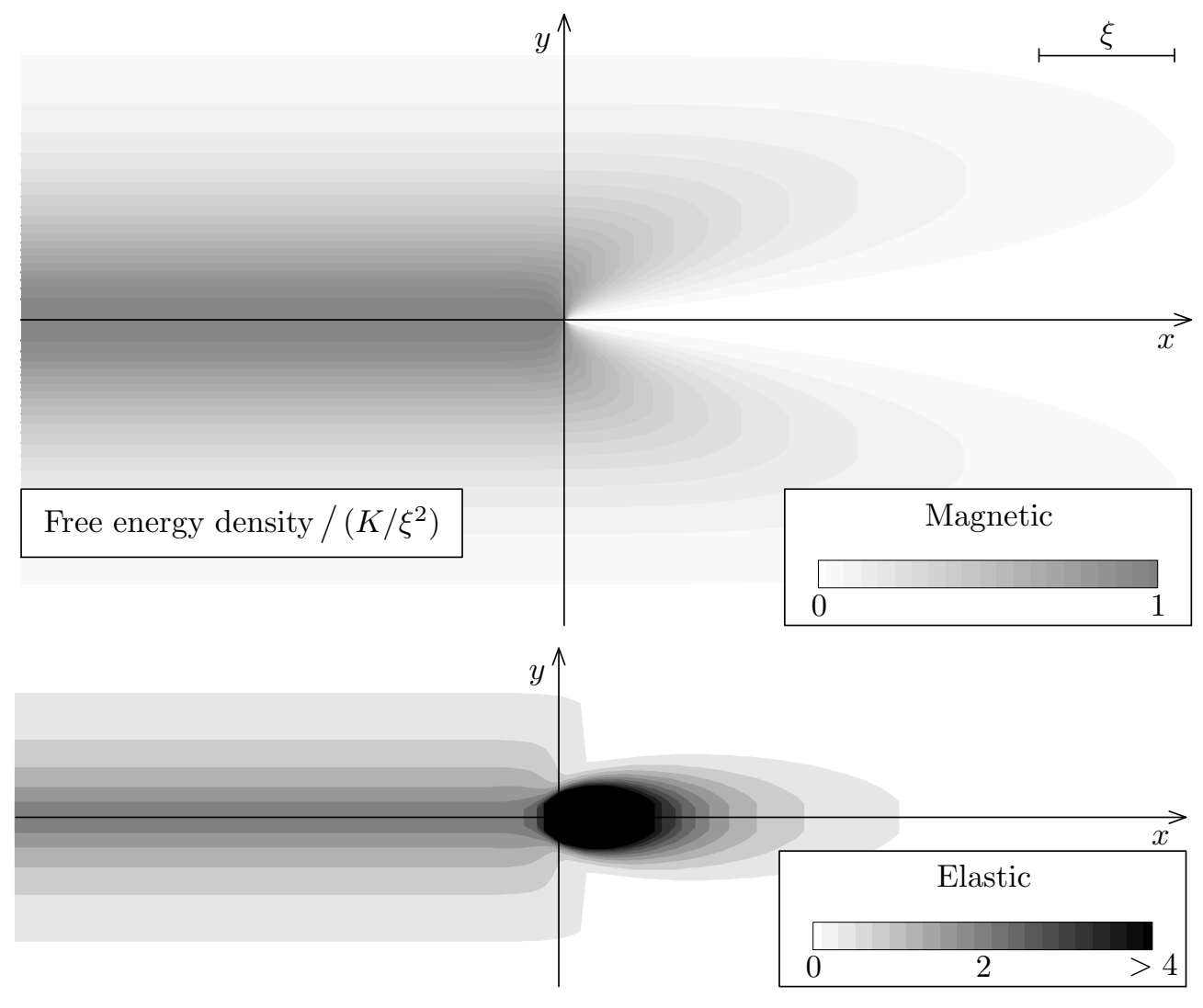

Figure 2: Density plots of the magnetic (upper graph) and elastic (lower) parts of the free-energy density, computed from the analytical solution (2.13). The elastic energy is localized close to the defect. On the contrary, the magnetic energy is mostly packed around the $\pi$-wall. The defect core is not symmetric with respect to the defect position. It is slightly shifted behind it, with respect to the direction of motion.

\subsection{Disclination velocity}

We have succeeded in finding a solution of the stationary Ginzburg-Landau equation for any value of $v$. We will now complete the eigenvalue analysis, and determine the correct disclination velocity, by imposing the global dissipation condition (2.1).

We have already noticed that, in the moving reference frame, time derivatives transform into spatial derivatives. Thus,

$$
\dot{\mathcal{F}}=v \int_{\mathcal{B}_{\circ}} \frac{\partial}{\partial x}\left(K|\nabla \Phi|^{2}+\chi_{\mathrm{a}} H^{2} \Phi^{2}\right) d x d y=v\left[\int_{\mathbb{R}}\left(K|\nabla \Phi|^{2}+\chi_{\mathrm{a}} H^{2} \Phi^{2}\right) d y\right]_{x=-\infty}^{x=+\infty} .
$$

When $|x|$ is very large, the principal value of the $q$-integral in (2.13) is dominated by the low $q$ values:

$$
\lim _{x \rightarrow \pm \infty} \frac{1}{4 \mathrm{i}} \mathrm{PV} \int_{\mathbb{R}} \frac{\mathrm{e}^{\mathrm{i} q x-k(q) y}}{q} d q=\frac{1}{4 \mathrm{i}} \lim _{x \rightarrow \pm \infty} \mathrm{PV} \int_{\mathbb{R}} \frac{\mathrm{e}^{\mathrm{i} q x-y / \xi}}{q} d q= \pm \frac{\pi}{4} \mathrm{e}^{-y / \xi}
$$

Thus,

$$
\dot{\mathcal{F}}=-\frac{\pi^{2} K v}{\xi^{2}} \int_{0}^{+\infty} \mathrm{e}^{-\frac{2 y}{\xi}} d y=-\frac{\pi^{2} K v}{2 \xi}
$$


The result (2.17) admits a simple physical interpretation, that already exhibits in (2.15). The quantity within square brackets in (2.15) is the free-energy contained in an infinite vertical strip of unit width, centered at $x$. When $x \rightarrow+\infty$, both the elastic and magnetic energy densities relax to 0 , as Figure 2 shows. As a consequence, the total energy stored in the right-side strip is negligible. The picture completely changes in the $x \rightarrow-\infty$ limit. A unit-width strip crossing the $\pi$-wall stores a finite amount of free energy, measured in (2.17). In fact, it is precisely the difference between the energies stored in those strips that keeps the defect moving. In the unit time, the defect motion replaces a left-side strip of width $v$, with free energy given in (2.17), with a right-side strip of equal width, with no free energy.

While the free energy variation depends only on the asymptotic structure of the director field, dissipation takes place in the whole domain. Indeed, it is so strong close to the moving defect, that we will be forced to exclude the core region in order to avoid an infinite dissipation. We have:

$$
\begin{aligned}
\mathcal{D} & =\gamma_{1} \int_{\mathbb{R}^{2}} \dot{\Phi}^{2} d x d y=\gamma_{1} v^{2} \int_{\mathbb{R}^{2}}\left(\frac{\partial \Phi}{\partial x}\right)^{2} d x d y \\
& =\frac{\gamma_{1} v^{2}}{16} \int_{\mathbb{R}^{2}} d x d y \mathrm{PV} \int_{\mathbb{R}} d q \mathrm{PV} \int_{\mathbb{R}} d q^{\prime} \mathrm{e}^{\mathrm{i}\left(q+q^{\prime}\right) x-\left(k(q)+k\left(q^{\prime}\right)\right)|y|} \\
& =\frac{\gamma_{1} v^{2}}{8} \mathrm{PV} \int_{\mathbb{R}} d q \mathrm{PV} \int_{\mathbb{R}} d q^{\prime} \frac{2 \pi \delta\left(q+q^{\prime}\right)}{k(q)+k\left(q^{\prime}\right)} \\
& =\frac{\pi \gamma_{1} v^{2}}{2} \int_{0}^{q_{\mathrm{M}} /(2 \pi)} \frac{d q}{\sqrt{q^{2}+\mathrm{i} q \lambda / \xi+1 / \xi^{2}}+\sqrt{q^{2}-\mathrm{i} q \lambda / \xi+1 / \xi^{2}}} \\
& =-\frac{\mathrm{i} \pi K v}{2 \xi} \int_{0}^{\xi / r_{\circ}}\left(\sqrt{1+\frac{\mathrm{i} \lambda}{s}+\frac{1}{s^{2}}}-\sqrt{1-\frac{\mathrm{i} \lambda}{s}+\frac{1}{s^{2}}}\right) d s,
\end{aligned}
$$

where $\delta$ denotes the Dirac delta function. The high- $q$ cutoff is needed in order to avoid the logarithmic divergence which the disclination induces both in the free energy (but not in its timederivative) and in the dissipation. This is related to the inverse of the core radius: $q_{\mathrm{M}}=2 \pi / r_{\circ}$.

When we replace (2.15) and (2.18) into the dissipation principle (2.1) we obtain the selfconsistency equation that determines $\lambda$ (i.e., $v$ ), as a function of the ratio $\xi / r_{0}$ :

$$
\int_{0}^{\xi / r_{\circ}}\left(\sqrt{1+\frac{\mathrm{i} \lambda}{s}+\frac{1}{s^{2}}}-\sqrt{1-\frac{\mathrm{i} \lambda}{s}+\frac{1}{s^{2}}}\right) d s=\mathrm{i} \pi
$$

The most interesting region in physical applications is $\xi \gg r_{0}$. The integral on the left hand side is dominated by its logarithmic high- $s$ divergence and we obtain:

$$
\mathrm{i} \lambda \log \frac{\xi}{r_{\circ}}=\mathrm{i} \pi \quad \Longrightarrow \quad v=\frac{2 \pi \sqrt{K \chi_{\mathrm{a}}}}{\gamma_{1} \log \left(\xi / r_{\circ}\right)}|H| \quad \text { when } \quad \xi \gg r_{\circ} .
$$

However, it is interesting to push the analysis of (2.19) into the opposite regime $\xi \ll r_{\circ}$. The low- $s$ terms dominate the integral on the left hand side and we find:

$$
\mathrm{i} \lambda \frac{\xi}{r_{\circ}}=\mathrm{i} \pi \quad \Longrightarrow \quad v=\frac{2 \pi \chi_{\mathrm{a}} r_{\circ}}{\gamma_{1}} H^{2} \quad \text { when } \quad \xi \ll r_{\circ} .
$$

The quite intriguing quadratic behaviour predicted by (2.21) must be handled carefully. When the external field becomes so intense that $\xi$ becomes of the order of $r_{\circ}$, we have to question our assumption of $r_{\circ}$ being independent from $\xi$. A more complete theory, that can be derived following the steps of [30, would yield an $r_{\circ}(\xi)$, and thus a disclination velocity depending only on the strength of the applied field.

Figure 3 shows the numerical solution of equation (2.19). The plot highlights that the transition between the two asymptotic regimes derived above occurs for $\xi \gtrsim r_{\circ}$. Even though this is the limit up to which we can seriously trust our analytical result, Figure 3 suggests that the nonlinear effects increase the disclination velocity. 


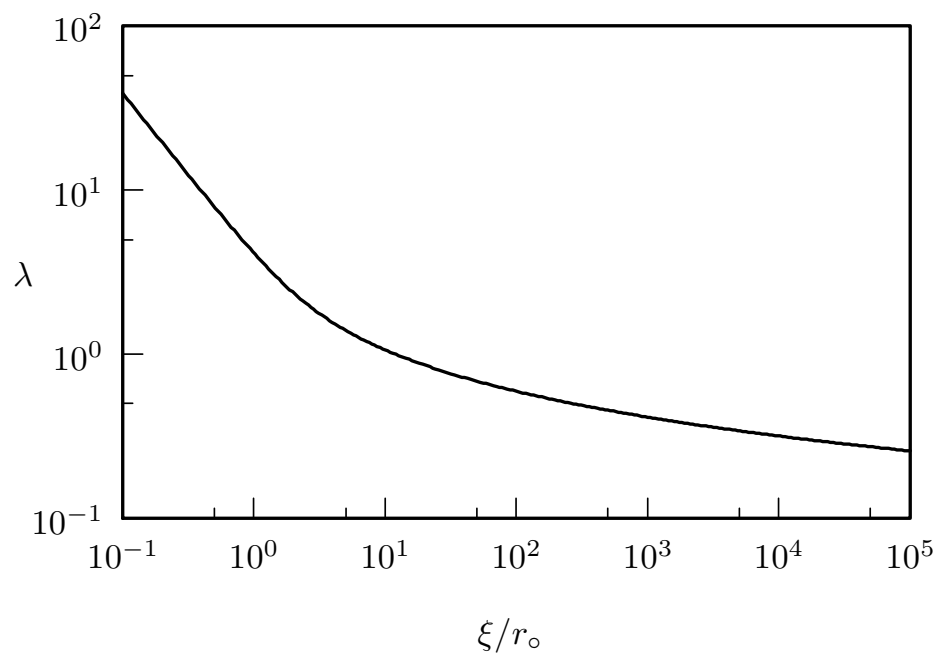

Figure 3: Disclination velocity as a function of the external applied field

\section{Defect interaction}

Let us now consider two nematic defects of opposite topological charges $s= \pm \frac{1}{2}$ placed on the $x$-axis at a distance $\Delta=2 d$, but in the absence of any external field. After a certain period of time these defects amalgamate and thus annihilate each other. The two cores disappear at once, with a large and rapid reduction in free-energy [13, 15, 26. Imagine, however, that we apply a magnetic field that favours the director orientation of the molecules that lie within the defects. The defect speed will be certainly reduced. In this section we show that it can be even reversed. It exists a critical distance such that the defect interaction is attractive only if their mutual distance is smaller than the critical one. Otherwise, they repel.

Throughout this section, we will work out in detail the geometry illustrated in Figure 4 The applied field lies parallel both to the line connecting the defects, and to the director orientation between them. In this geometry, the defects may only approach or separate, so avoiding but more complicated motions such as mutual rotations.

We compute the speed of two stationarily moving defects. We will show below that the stationarity assumption holds approximately even when the defect distance becomes of the order of, or smaller than, the magnetic coherence length. It must certainly be abandoned when one wants to describe the complete annihilation process, and in particular when the defect distance becomes of the order of the core radius.

In the final subsection we will briefly analyse the case when the external field determines a generic angle $\alpha$ with respect to the defect line. In this case the motion may be much more complicated. However, we are able to estimate how the nature of the defect interaction depends on $\alpha$. More precisely, we will determine for which values of $\alpha$ the defect interaction may become repulsive, and how the critical distance depends on the external field direction.

The time-dependent Ginzburg-Landau equation (2.6) is linear, due to the parabolic approximation we used for the magnetic energy. We can thus obtain a solution describing two stationarily approaching (or separating) defects by simply superposing two functions of the type (2.13). More precisely, we add a solution describing a defect placed at $x=+d$, travelling with velocity $-v$, and a solution describing a defect placed at $x=-d$, travelling with velocity $+v$. The velocity $v(d)$ 


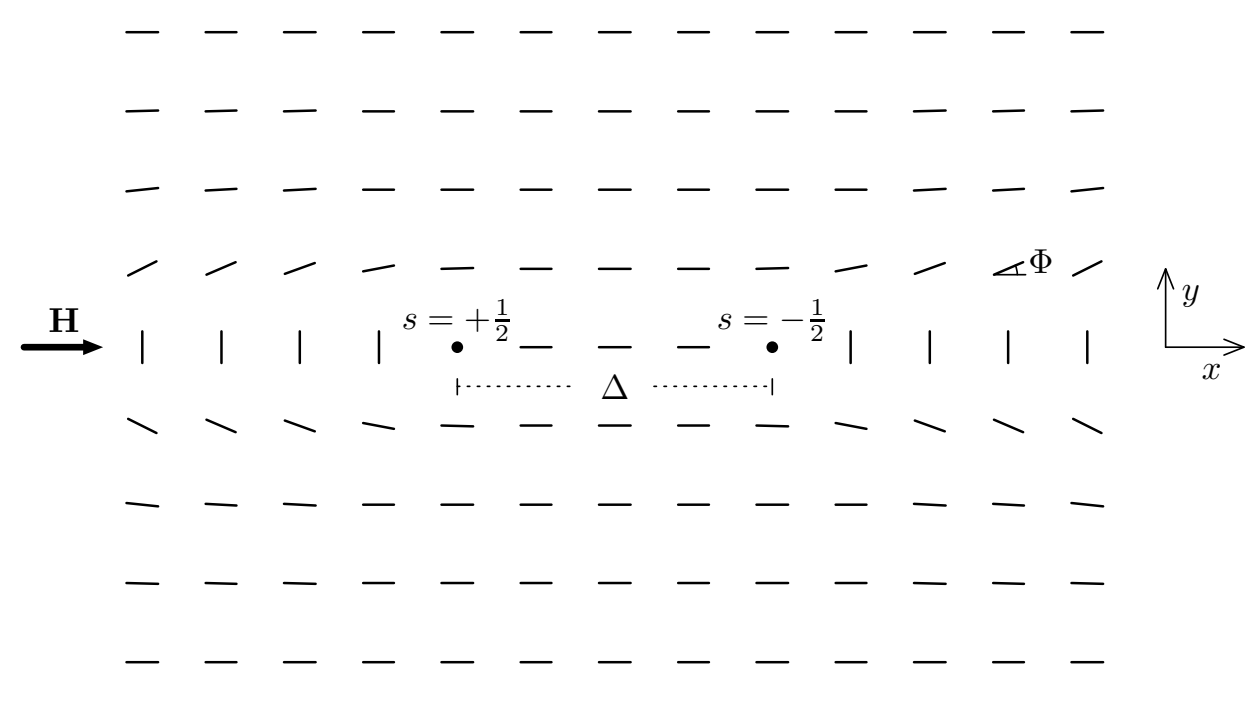

Figure 4: Two attracting defects placed at a distance $\Delta$ along the $x$-axis. The external field is parallel both to the line connecting the defects and to the director orientation between them.

will be determined later, again using the dissipation principle. The director field is given by:

$$
\begin{aligned}
\Phi(x, y) & =\varepsilon(y)\left[\frac{\pi}{2} \mathrm{e}^{-\frac{|y|}{\xi}}+\frac{1}{4 \mathrm{i}} \mathrm{PV} \int_{\mathbb{R}} \frac{\mathrm{e}^{\mathrm{i} q(x-d)-k(q)|y|}-\mathrm{e}^{\mathrm{i} q(x+d)-k(-q)|y|}}{q} d q\right] \\
& =\varepsilon(y)\left[\frac{\pi}{2} \mathrm{e}^{-\frac{|y|}{\xi}}-\int_{0}^{+\infty} \cos q x \sin (q d+\operatorname{Im} k(q)|y|) \mathrm{e}^{-\operatorname{Re} k(q)|y|} \frac{d q}{q}\right] .
\end{aligned}
$$

Figure $[5$ illustrates the countervailing tendencies of the elastic and magnetic energies: the elastic contribution aims at annihilating the defects in order to relax the infinite core energy. On the contrary, the magnetic field tries to broaden the intermediate region, where all the molecules are already correctly aligned.

The free energy associated with the traveling configuration (3.1) is given by:

$$
\mathcal{F}(\Delta, \lambda)=K \pi\left[\frac{\pi L}{\xi}+\operatorname{arcsinh} \frac{\xi}{r_{\circ}}-K_{0}\left(\frac{\Delta}{\xi}\right)-\frac{\pi \Delta}{\xi}+2 \int_{0}^{\infty} \frac{\sin ^{2} \frac{\Delta s}{2 \xi}}{s^{2} \sqrt{1+s^{2}}} d s+F_{1}(\Delta, \lambda)+F_{2}(\lambda)\right] .
$$

The first term in (3.2) diverges in the $L \rightarrow+\infty$ limit ( $L$ is the horizontal scale of the system: $x \in[-L, L]$ ). This term corresponds to the energy of the two walls. The second term is related to the infinite elastic energy stored in the defects $(r$ o is the core radius as in the preceding section). The next three terms depend only on the defect distance, but not on their velocity. In particular, the term containing the modified Bessel function of the second kind $K_{0}$ is able to cancel the free energy divergence when $\Delta \simeq r_{\circ}$. Finally, both $F_{1}$ and $F_{2}$ are velocity corrections, which vanish if 


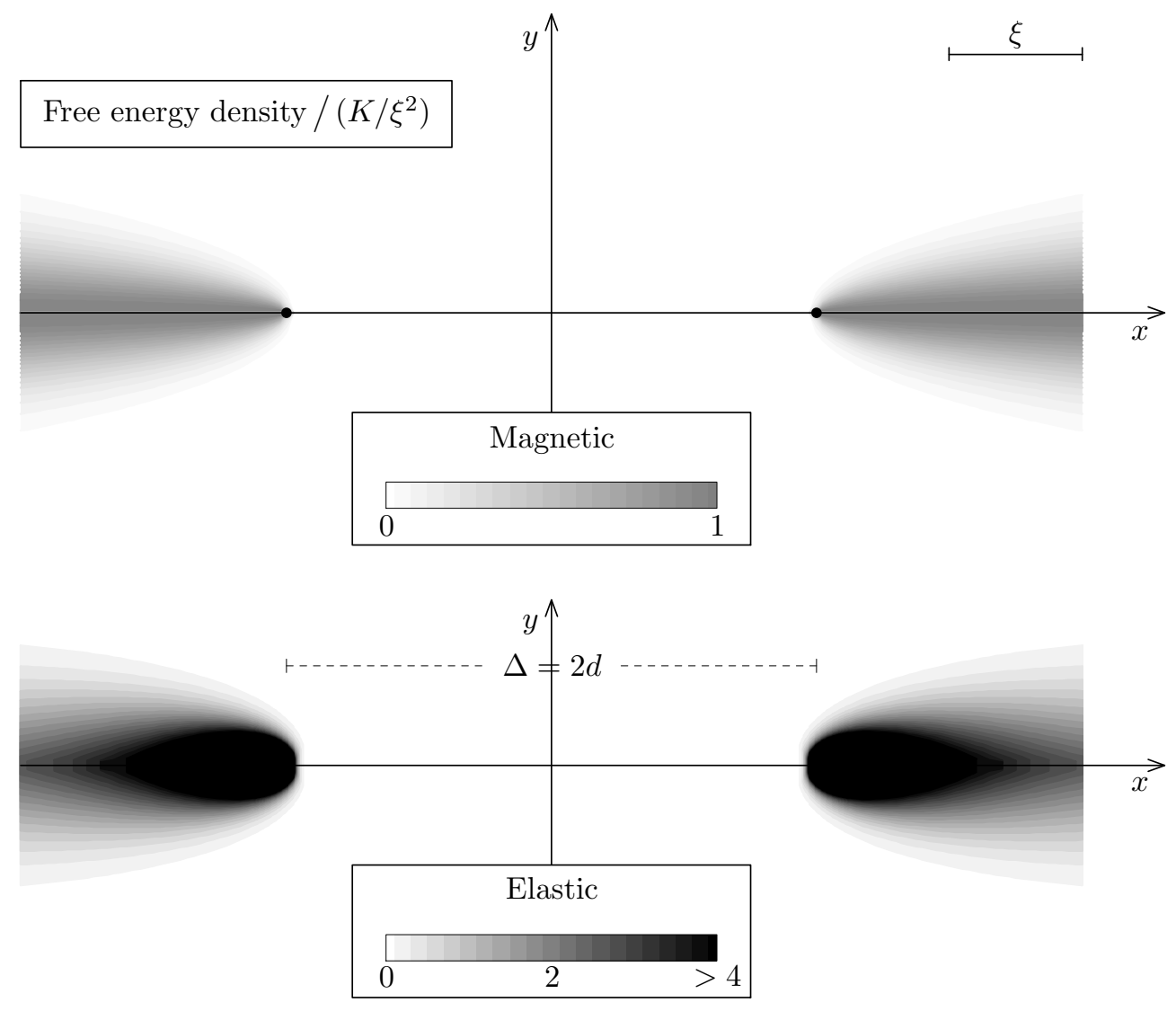

Figure 5: Density plot of the magnetic free-energy density (upper graph) and the elastic freeenergy density (lower graph), computed from the analytical solution (3.1). For these particular values of the distance and the magnetic coherence length, it will turn out that the defects are separating (that is, $v<0$ ). Both the magnetic and the elastic free-energy densities are mainly concentrated on the defect walls. Between the defects, the free-energy density is negligible.

$\lambda(i . e ., v)$ vanishes:

$$
\begin{aligned}
F_{1}(\Delta, \lambda) & :=\frac{1}{2} \int_{0}^{\infty} \operatorname{Re}\left[\left(\mu(\lambda, s)-\mu(0, s)+\frac{1}{\mu(\lambda, s)}-\frac{1}{\mu(0, s)}\right)\left(1-\mathrm{e}^{-\mathrm{i} s \Delta / \xi}\right)\right] \frac{d s}{s^{2}} \\
& -\frac{1}{2} \int_{0}^{\infty} \operatorname{Re}\left[\left(\frac{1}{\mu(\lambda, s)}-\frac{1}{\mu(0, s)}\right) \mathrm{e}^{-\mathrm{i} s \Delta / \xi}\right] d s \\
F_{2}(\lambda) & :=\int_{0}^{\infty}\left(\frac{1}{\mu(\lambda, s)+\mu(-\lambda, s)}-\frac{1}{2 \mu(0, s)}\right) d s \\
& -\frac{1}{4} \int_{0}^{\infty} \frac{(\mu(\lambda, s)-\mu(-\lambda, s))^{2}}{\mu(\lambda, s)+\mu(-\lambda, s)}\left(1+\frac{1}{\mu(\lambda, s) \mu(-\lambda, s)}\right) \frac{d s}{s^{2}}
\end{aligned}
$$

where $\mu(s)$ is the positive-real-part square-root of $\mu^{2}(s)=1+\mathrm{i} s \lambda+s^{2}$, and $\lambda=\frac{\gamma_{1}}{2 \sqrt{K \chi_{\mathrm{a}}}} \frac{v}{|H|}$ as in the preceding section.

In the absence of backflow, the dissipation stems only from the director rotation. When computing $\dot{\Phi}$, we assume that only the defect position $d$ depends on time. In our stationary approximation, we thus neglect the time derivative of the velocity $v$. However, we remark that 
when we want to determine the non-stationary effects depending on $\dot{v}$, we are no longer allowed to use the solution (3.1). The dissipation function is given by:

$$
\mathcal{D}=\gamma_{1} \int_{\mathbb{R}^{2}} \dot{\Phi}^{2} d x d y=\frac{\pi \dot{d}^{2}}{2}\left[\operatorname{arcsinh} \frac{\xi}{r_{\circ}}+K_{0}\left(\frac{\Delta}{\xi}\right)+G_{1}(\Delta, \lambda)+G_{2}(\lambda)\right],
$$

with

$$
\begin{aligned}
G_{1}(\Delta, \lambda) & :=\int_{0}^{\infty} \operatorname{Re}\left[\left(\frac{1}{\mu(\lambda, s)}-\frac{1}{\mu(0, s)}\right) \mathrm{e}^{-\mathrm{i} s \Delta / \xi}\right] d s \quad \text { and } \\
G_{2}(\lambda) & :=2 \int_{0}^{\infty}\left(\frac{1}{\mu(\lambda, s)+\mu(-\lambda, s)}-\frac{1}{2 \mu(0, s)}\right) d s
\end{aligned}
$$

The functions $G_{1}$ and $G_{2}$ vanish in the low-velocity limit $v \rightarrow 0$. The dissipation principle delivers the self-consistency equation that determines the defect velocity $v=-\dot{d}$ :

$$
\begin{aligned}
& \frac{\partial \mathcal{F}}{\partial d} \dot{d}+\mathcal{D}=0 \Longleftrightarrow \\
& K_{1}\left(\frac{\Delta}{\xi}\right)+\frac{\Delta}{\xi} \int_{0}^{\infty} \frac{\sin s d s}{s \sqrt{\frac{\Delta^{2}}{\xi^{2}}+s^{2}}}+\xi \frac{\partial F_{1}}{\partial \Delta}-\frac{\lambda}{2}\left[\operatorname{arcsinh} \frac{\xi}{r_{\circ}}+K_{0}\left(\frac{\Delta}{\xi}\right)+G_{1}(\Delta, \lambda)+G_{2}(\lambda)\right]=\pi .
\end{aligned}
$$

Before analyzing the solutions $\lambda(\Delta)$ of equation (3.8), we want to stress the importance of the first two terms in its left-hand side. In fact, if we define

$$
f(x):=K_{1}(x)+x \int_{0}^{\infty} \frac{\sin s}{s \sqrt{x^{2}+s^{2}}} d s
$$

we have:

$$
\left.\frac{\partial \mathcal{F}}{\partial \Delta}\right|_{v=0}=\frac{K \pi}{\xi}\left[f\left(\frac{\Delta}{\xi}\right)-\pi\right]
$$

Thus, in general, the defects will approach or separate depending on whether $f(\Delta / \xi)$ exceeds $\pi$ or not. We postpone the analysis of the properties of $f$ to the next subsection, when we will generalize equation (3.10) to the case of tilted applied fields. As for now, we only remark that $f(x)=\pi$ when $x=x_{\mathrm{cr}} \doteq 0.377388$. The function $f$ is greater than $\pi$ (thus inducing defect attraction) when $\Delta<x_{\mathrm{cr}} \xi$. Defect repulsion is induced at distances greater than $x_{\mathrm{cr}} \xi$.

Figure [6 illustrates the numerical solutions of equation (3.8) for three different values of the ratio between the magnetic coherence length and the core radius. They exhibit the following properties.

- In the large distance limit, all Bessel functions decay exponentially with $\Delta / \xi$. The functions $G_{1}, G_{2}$, and the derivative of $F_{1}$ vanish too. Furthermore,

$$
\lim _{x \rightarrow \infty} x \int_{0}^{\infty} \frac{\sin s}{s \sqrt{x^{2}+s^{2}}} d s=\int_{0}^{\infty} \frac{\sin s}{s} d s=\frac{\pi}{2} .
$$

Thus, the large-distance limit of $\lambda$ is given by

$$
\lim _{\Delta \rightarrow \infty} \lambda(\Delta)=-\frac{\pi}{\operatorname{arcsinh}\left(\xi / r_{\circ}\right)} \quad \Longrightarrow \quad v \simeq-\frac{2 \pi \sqrt{K \chi_{\mathrm{a}}}|H|}{\gamma_{1} \operatorname{arcsinh}\left(\xi / r_{\circ}\right)} \quad \text { when } \Delta \gg \xi .
$$

The defects repel, and move at a constant speed. In fact, if we compare (3.12) with (2.20), we notice that in the large-distance limit the defects behave independently, each moving at the velocity computed in the 1 -defect case, $\operatorname{since} \operatorname{arcsinh}\left(\xi / r_{\circ}\right) \simeq \log \left(\xi / r_{\circ}\right)$ when $\xi \gg r_{\circ}$.

- The critical distance at which the defect interaction changes sign does not depend on the core radius $r_{\mathrm{o}}$ : all plots in Figure 6 cross the $x$-axis at $\Delta=x_{\mathrm{cr}} \xi$. 
- In the small distance limit, the stationary approximation we have used is not well justified. In this limit, the velocity diverges, and all terms depending on $\dot{v}$ must be taken into account in the equation of motion. In any case, we remark that the Bessel function $K_{1}$ induces a divergence in the derivative of the free-energy that scales as the inverse of the defect distance. Figure [ suggests that the fully nonlinear regime is limited to distances much smaller than the magnetic coherence length.

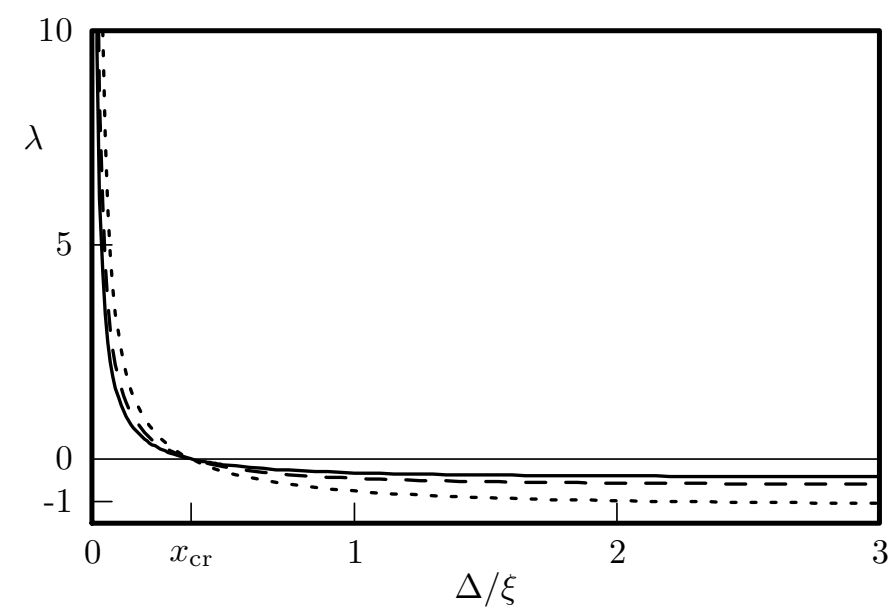

Figure 6: Defect velocity as a function of the external field for different values of the core radius $r_{\circ}: \xi / r_{\circ}=10$ (dotted line), 100 (dashed), 1000 (full). For any value of the core radius, the defects attract only if their distance is smaller than $\Delta_{\text {cr }}$.

\subsection{Tilted external fields}

Let us now imagine that the external field is rotated of an angle $\alpha$ with respect to the direction of Figure 4 In this case, the nematic director is forced to relax to the external field direction everywhere but on the topologically irreducible $\pi$-wall. The motion will be much more complex - the defects will rotate, and the $\pi$-wall will not necessarily be straight at all times. However, it is possible to ascertain whether the defect interaction will lead to attraction or repulsion. To this aim, we imagine to pin the defects at a distance $\Delta$, and we look for the stationary director field in the presence of a tilted external field. Then, we compute the free-energy of the stationary solution, and we check the sign of its derivative with respect to the distance. We stress that it is not possible to use this derivative in a dissipation principle to obtain a defect velocity. However, its sign will determine whether or not the defects, whatever their complex motion, will approach each other.

The stationary director field can be simply derived by running through the above steps again. In the presence of two defects placed at $x= \pm d$, and an external field tilted of an angle $\alpha$ with respect to the $x$-axis, the stationary configuration is given by:

$$
\Phi(x, y)=\varepsilon(y)\left[\alpha+\left(\frac{\pi}{2}-\alpha\right) \mathrm{e}^{-\frac{|y|}{\xi}}-\int_{0}^{+\infty} \cos q x \sin q d \mathrm{e}^{-k_{0}(q)|y|} \frac{d q}{q}\right]
$$

where $k_{0}(q)$ coincides with the zero-velocity limit of $k(q)$ above: $\quad k_{0}(q):=\sqrt{q^{2}+1 / \xi^{2}}$.

If we compute the free-energy associated to (3.13), and we differentiate it with respect to $\Delta$, we obtain the generalization of equation (3.10):

$$
\left.\frac{\partial \mathcal{F}}{\partial \Delta}\right|_{v=0}=\frac{K \pi}{\xi}\left[f\left(\frac{\Delta}{\xi}\right)-(\pi-2 \alpha)\right] .
$$

with the same $f$ defined in (3.9). The left panel of Figure 7 shows the plot of $f$. It enjoys the following properties: 
- $f^{\prime}(x)=\frac{1}{2}\left(K_{0}(x)-K_{2}(x)\right)<0$ for all $x>0$. Thus, the critical distance at which the defect interaction becomes repulsive increases when the external field is tilted. Furthermore, the free energy is a concave function of the distance between the defects.

- $\lim _{x \rightarrow 0} f(x)=+\infty$ and $\lim _{x \rightarrow \infty} f(x)=\frac{\pi}{2}$. More precisely,

$$
f(x)=\frac{1}{x}-\frac{x}{2} \log x+O(x) \text { as } x \rightarrow 0^{+} \quad \text { and } \quad f(x)=\frac{\pi}{2}+o\left(\mathrm{e}^{-x}\right) \text { as } x \rightarrow+\infty .
$$

Thus, the equation

$$
\frac{\partial \mathcal{F}}{\partial \Delta}=0 \quad \Longleftrightarrow \quad f(x)=\pi-2 \alpha,
$$

which determines the equilibrium distances, possesses exactly one solution if and only if $\alpha<\frac{\pi}{4}$. This limiting value for the tilting angle could be easily predicted. In fact, if the external field determines an angle greater than $\frac{\pi}{4}$ with the defect line, the director orientation between the defects costs more magnetic energy than the director orientation outside the defects. Thus, in this case, the external field strengthens the defect attraction at any distance.

In summary:

- When $\alpha=0$, the defects attract if $\Delta<\Delta_{\text {cr }}\left(\frac{\pi}{2}\right)=0.3774 \xi$; they repel when $\Delta>\Delta_{\text {cr }}\left(\frac{\pi}{2}\right)$.

- When $\alpha>0$, the critical distance $\Delta_{\text {cr }}(\beta)$ increases. It diverges when $\alpha \rightarrow \frac{\pi}{4}^{-}$.

- When $\alpha \geq \frac{\pi}{4}$ there is no critical distance: the defects always attract (the external field enhances the attraction).
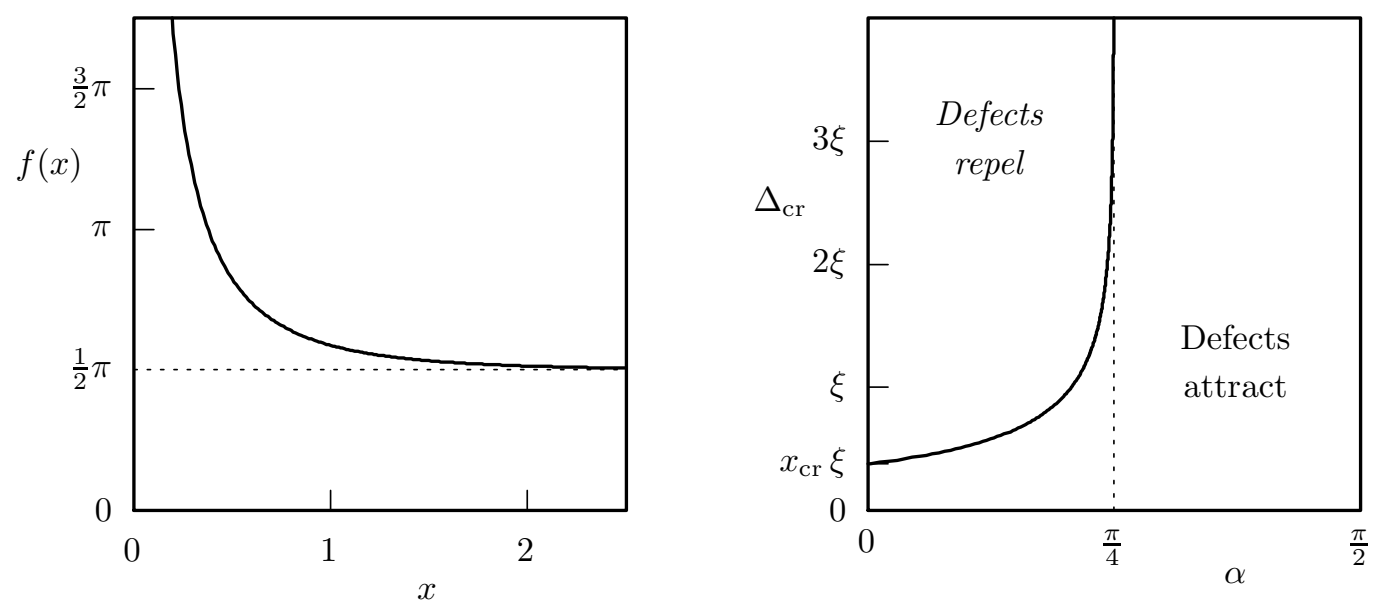

Figure 7: Left: Plot of the function $f$ defined in the text. Right: Critical value of the defect distance as a function of the tilt angle $\alpha$.

\section{Discussion}

We have studied the motion of a single disclination, and a disclination dipole in an external field. Our results show that it is possible to drive the disclinations by suitably adjusting the external field direction and strength.

In the case of a single disclination we have shown that the disclination velocity depends almost linearly on the field strength, since the coefficient depends on the logarithm of the field strength 
(see equation (2.20) $)$. Figure 3 shows that the linear scaling is abandoned when $\xi \simeq r_{\circ}$. Equation (2.21) shows that below this regime the disclination velocity is expected to scale as the square of the applied strength. However, this last prediction should be tested carefully, since it is surely influenced by our assumption that the core radius does not depend on the field strength.

We have also computed the velocity of two opposite disclinations in the presence of a field that promotes the director orientation between them. The velocity depends on the defect distance $\Delta$ but, whatever the value of the ratio $\xi / r_{\circ}$, they defects approach if $\Delta$ is smaller that a critical distance $\Delta_{\mathrm{cr}}$; otherwise, they repel. We have finally generalized our calculations in order to deal with rotated external fields. Figure 7 (right) shows how the $\Delta_{\text {cr }}$ depends on the angle $\alpha$ that the external field determines with the lie which connects the defects.

The introduction of backflow may change the picture we have developed, both in the one and two-disclination cases. In the one disclination case the dissipation may sometimes be significantly reduced. The system can match the disclination motion with a flow that almost cancels the dissipation in the crucial core region. Whether this occurs seems likely to depend on the charge of the central disclination. We note that central physical and mathematical issues associated with backflow in nematic liquid crystal problems still remain open even over a quarter of a century after its essential role was first realized [20].

Likewise in the two-disclination problem, our computed defect velocity is symmetrical in both disclinations. This is also an effect of the no-backflow simplification. A generalization of the present study should allow to compute the different approaching or separating velocities. It could even happen that one defect moves towards the other, but that the other retreats faster still, allowing the defects eventually to separate. However, the existence of a critical distance that reverses the defect interaction can not be erased by backflow effects. The critical distance stems from the balance between the elastic and magnetic free-energy gains. The magnetic gain does not depend on the defect distance, while the elastic gain vanishes when the defects move apart. Thus, at some intermediate distance one overwhelms the other.

\section{Acknowledgements}

This work has been supported by a British Council Anglo-Italian collaborative grant. P.B. acknowledges the hospitality of the University of Southampton, where this work was carried out.

\section{References}

[1] F.C. Frank. On the theory of liquid crystals. Discuss. Far. Soc. 25 (1958), 19-28.

[2] N.D. Mermin: The topological theory of defects in ordered media. Rev. Mod. Phys. 51 (1979), 591-648.

[3] M. KlÉman: Defects in liquid crystals. Rep. Prog. Phys. 52 (1989), 555-654.

[4] P. Biscari \& G. Guidone Peroli: A hierarchy of defects in biaxial nematics. Commun. Math. Phys. 186 (1997), 381-392.

[5] G. Guidone Peroli \& E.G. Virga: Nucleation of topological dipoles in nematic liquid crystals. Commun. Math. Phys. 200 (1999), 195-210.

[6] P.E. Cladis \& M. KlÉman: Non-singular disclinations of strength $S=1$ in nematics. J. Phys. (Paris) 33 (1972), 591-598.

[7] C.W. Oseen. The theory of liquid crystals Trans. Far. Soc. 29 (1933), 883-900.

[8] H. Zocher. The effect of a magnetic field on the nematic state. Trans. Far. Soc. 29 (1933), 945-957. 
[9] P.G. De Gennes and J. Prost. The Physics of Liquid Crystals, Ed. Clarendon Press, Oxford (1993).

[10] J.L. ERICKSEn. Liquid crystals with variable degree of orientation. Arch. Rat. Mech. Anal. 113 (1989), 97-120.

[11] F.M. LesLiE: Continuum theory for nematic liquid crystals. Cont. Mech. Thermodyn. 4 (1992), 167-175.

[12] G. Ryskin \& M. KRemenetsky: Drag force on a line defect moving through an otherwise undisturbed field: disclination line in a nematic liquid crystal. Phys. Rev. Lett. 67 (1991), 1574-1577.

[13] A. Pargellis, N. Turok \& B. Yurke: Monopole-antimonopole annihilation in a nematic liquid crystal. Phys. Rev. Lett. 67 (1991), 1570-1573.

[14] L.M. Pismen \& B.Y. Rubinstein: Motion of interacting point defects in nematics. Phys. Rev. Lett. 69 (1992), 96-99.

[15] C. Denniston: Disclination dynamics in nematic liquid crystals. Phys. Rev. B 54 (1996), $6272-6275$.

[16] G. Guidone Peroli \& E.G. Virga: Annihilation of point defects in nematic liquid crystals. Phys. Rev. E 54 (1996), 5235-5241.

[17] G. Guidone Peroli and E.G. Virga: Dynamics of point defects in nematic liquid crystals. Physica D 111 (1998), 356-372.

[18] P. Biscari, G. Guidone Peroli, E.G. Virga: A statistical study for evolving arrays of nematic point defects. Liquid Crystals 26 (1999), 1825-1832.

[19] A.N. Pargellis, P. Finn, J.W. Goodby, P. Panizza, B. Yurke \& P.E. Cladis: Defect dynamics and coarsening dynamics in smectic-C films. Phys. Rev. A 46 (1992), 7765-7776.

[20] M. G. Clark \& F. M. Leslie: A calculation of orientational relaxation in nematic liquid crystals. Proc. R. Soc. Lond. A 361 (1978), 463-485.

[21] P.D. Olmsted \& P. Goldbart: Theory of the nonequilibrium phase transition for nematic liquid crystals under shear flow. Phys. Rev. A 41 (1990), 4578-4581.

[22] P.D. Olmsted \& P. Goldbart: Isotropic-nematic transition in shear flow: State selection, coexistence, phase transitions, and critical behaviour. Phys. Rev. A 46 (1992), 4578-4993.

[23] G. Richardson: Line disclination dynamics in uniaxial nematic liquid crystals. Q. Jl. Mech. Appl. Math. 53 (2000), 49-71.

[24] C. Denniston, E. Orlandini \& J.M. Yeomans: Simulations of liquid crystal hydrodynamics in the isotropic and nematic phases. Europhys. Lett. 52 (2000), 481-487.

[25] C. Denniston, E. Orlandini \& J.M. Yeomans: Lattice Boltzmann simulations of liquid crystal hydrodynamics. Phys. Rev. E 63 (2001), 056702.

[26] D. SvenšEK \& S. Žumer: Hydrodynamics of pair-annihilating disclination lines in nematic liquid crystals. Phys. Rev. E 66 (2002), 021712.

[27] G. Tóth, C. Denniston \& J.M. Yeomans: Hydrodynamics of topological defects in nematic liquid crystals. Phys. Rev. Lett. 88 (2002) 105504.

[28] H. Brezis, J.M. Coron, and E. Lieb. Harmonic maps with defects. Comm. Math. Phys. 107 (1986), 649-705. 
[29] N. Schopohl and T.J. Suuckin. Defect core structure in nematic liquid crystals. Phys. Rev. Lett. 59 (1987), 2582-2584.

[30] P. Biscari \& T.J. Sluckin: Expulsion of disclinations in nematic liquid crystals. Euro. Jnl. Appl. Math. 14 (2003), 39-59.

[31] P. Cermelli and E. Fried. The evolution equation for a disclination in a nematic liquid crystal. Proc. R. Soc. London A 458 (2002), 1-20. 